\begin{tabular}{|c|c|}
\hline Title: & $\begin{array}{l}\text { DQ0 Modelling and Parameterization of small Delta connected PM Synchronous } \\
\text { Machines }\end{array}$ \\
\hline Authors: & $\begin{array}{l}\text { Simon Decker, Simon Foitzik, Felix Rehm, Matthias Brodatzki, Christoph Rollbuhler, } \\
\text { Johannes Kolb, Michael Braun }\end{array}$ \\
\hline Institute: & $\begin{array}{l}\text { Karlsruhe Institute of Technology (KIT) } \\
\text { Institute of Electrical Engineering (ETI) }\end{array}$ \\
\hline Type: & Conference Proceedings \\
\hline Published at: & $\begin{array}{l}2020 \text { International Conference on Electrical Machines (ICEM), Göteborg, Sweden, } \\
2020 \\
\text { Publisher: IEEE } \\
\text { Year: } 2020 \\
\text { ISBN: } 978-1-72819-945-0\end{array}$ \\
\hline Hyperlinks: & https://doi.org/10.1109/ICEM49940.2020.9270801 \\
\hline
\end{tabular}

(c) 2020 IEEE. Personal use of this material is permitted. Permission from IEEE must be obtained for all other uses, in any current or future media, including reprinting/republishing this material for advertising or promotional purposes, creating new collective works, for resale or redistribution to servers or lists, or reuse of any copyrighted component of this work in other works. 


\title{
DQ0 Modelling and Parameterization of small Delta connected PM Synchronous Machines
}

\author{
Simon Decker, Simon Foitzik, Felix Rehm, Matthias Brodatzki, Christoph Rollbühler, \\ Johannes Kolb, Michael Braun
}

\begin{abstract}
This paper presents an extended dq0-model for small delta-connected Permanent Magnet Synchronous Machines (PMSM), the design of a prototype and the parameterization of the model parameters by testbench measurement. The familiar dq-fundamental equations are thereby extended to consider harmonic effects. This allows the inclusion of the zero-sequence flux-linkage. The model, based on the dq0-flux-linkages and the stator resistance, enables the calculation of the zero-sequence current and a more precise inner torque estimation compared to state of the art fundamental models. The rotor position dependent dq-flux-linkage estimation is based on the measured dq-voltages and the solution of the simplified differential system equation. Detection of the zerosequence current yields to the zero-sequence flux-linkage. In this paper, we also present a prototype design of a PMSM machine with additional zero-sequence current sensing. Testbench measurement at constant controlled currents enables the parameter identification. For validation, the identified parameters are compared with existing Finite Element Analysis.
\end{abstract}

Index Terms - Electric machines, delta connection, Fourier analyses, parameter extraction, permanent magnet machines, system identification, system modelling, zero-sequence current.

\section{INTRODUCTION}

$\mathrm{S}$ mall electric drives with power from a few hundred watts up to one kilo watt, developed for car actuators, drivetrains, electric bikes or scooters, are nowadays often realized as PMSM. For mass production, these machines are designed with concentrated- and delta-connected stator windings. The nonlinear magnetic circuit, spatial harmonics and the zero-sequence flux- and current-component yield to additional losses, desaturation effects and additional torque ripple [1]. State of the art equivalent circuit models are fundamental dq-models considering saturation based on mean values, without rotor position dependencies, as shown in [2]. Therefore, in the past the delta-connected PMSMs were often described and analyzed as converted star connected PMSMs by wye/delta- transformation. The different electromagnetic behaviour concerning the flux-linkages as well as the missing zero-sequence components, leads to less precise and possibly incorrect model descriptions [3]. But the quality of model based control algorithms as well as precise system simulation rely on a detailed model descriptions and their parameters.

S. Decker is with Karlsruhe Institute of Technology, 76131 Karlsruhe, Germany (e-mail: simon.decker@kit.edu, (D) https ://orcid.org/0000-00018185-2309).

S. Foitzik is with Karlsruhe Institute of Technology, 76131 Karlsruhe, Germany (e-mail: simon.foitzik@kit.edu).

F. Rehm is with Karlsruhe Institute of Technology, 76131 Karlsruhe, Germany (e-mail: felix.rehm@kit.edu).

M. Brodatzki is with Karlsruhe Institute of Technology, 76131 Karlsruhe, Germany (e-mail: matthias.brodatzki@kit.edu).

C. Rollbühler is with Karlsruhe Institute of Technology, 76131 Karlsruhe, Germany (e-mail: c.rollbuehler@kit.edu).

J. Kolb is with SHARE at KIT, 76131 Karlsruhe, Germany (e-mail: johannes.kolb@schaeffler.com).

M. Braun is with Karlsruhe Institute of Technology, 76131 Karlsruhe, Germany (e-mail: michael.braun@kit.edu).
Due to slotting and other design parameters of electrical machines, torque distortion is unpreventable. But precise machine models filled with flux-linkage lookup tables based on the currents, the rotor speed and the rotor position enable modern inner torque / or noise distortion compensation algorithms. Smooth output torque can, for example, achieved with rotor position depended torque reference lookup tables, Fig. 1.

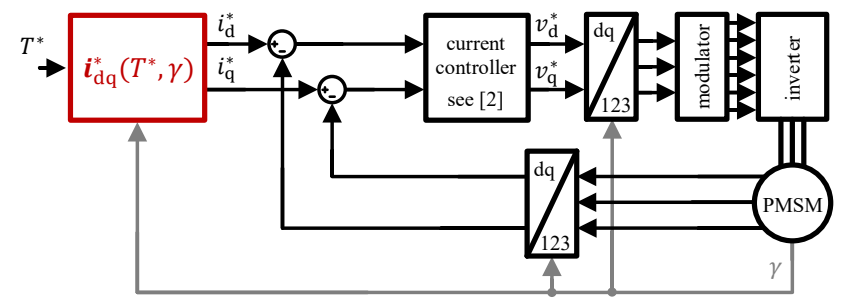

Fig. 1 Control strategy and MTPA/MTPV rotor position and reference torque lookup tables (in red) for the dq-currents and smooth output torque of the machine.

Moreover, for model based self-sensing [4] and fault detection algorithms of delta-connected PMSMs precise models with the knowledge of also the zero-sequence components, even in simulation, are mandatory.

Several models and identification methods were presented in the past. In [5], an extended model is derived with position dependent flux-linkages and incremental inductances for a precise description of the inner torque ripple at constant and various currents. For parameter identification of this model a simplified approach is used due to the current distortion at testbench control. In [6], an extended model of the inner torque of star-connected PMSMs based on dq-flux-linkages is described. Testbench measurements of the permanent magnet flux-linkage are done at steady-state with harmonic analysis of the back-EMF. Additional, locked-rotor tests enable calculation of differential inductances of each rotor position. Compared to the aforementioned methods of modelling and parameterization, this paper shows the parameter identification of position dependent flux-linkages only by steady-state testing at constant currents at different rotor speeds. With this parameterized dq0-flux-linkages, the precise inner torque and the zero-sequence current at any time can be calculated. The build-up and FEA analysis of the fluxlinkages of the investigated PMSM Device Under Test (DUT) is shown. Furthermore, the additional zero-sequence current sensing is motivated and the implementation into the phase interconnector is displayed. With the implemented repetitive control algorithm, constant controlled currents can be applied and enable the parameter identification. The necessary parameterization algorithm is thereby briefly explained and implemented in the post processing of the measured data. 


\section{DQ0 - MODEL}

The dq0-model of the equivalent circuit is shown in Fig. 2 and derived in the following. We assume three symmetric, delta-connected stator windings. For simplification, the friction as well as the iron losses are neglected. Furthermore, dielectric currents, thermal, skin and proximity effects are not considered.

The machines three phase stator voltage equations are derived from Ohm's law, Kirchhoff's law and the Maxwell's equations. Park-transformation, including the zero-sequence component [6], from the stator-oriented three phase system to the rotor-oriented dq0-reference frame yields the equations (1) to (3).

$$
\begin{gathered}
v_{\mathrm{d}}=R i_{\mathrm{d}}+\frac{\mathrm{d} \psi_{\mathrm{d}}}{\mathrm{d} t}-\omega \psi_{\mathrm{q}} \\
v_{\mathrm{q}}=R i_{\mathrm{q}}+\frac{\mathrm{d} \psi_{\mathrm{q}}}{\mathrm{d} t}+\omega \psi_{\mathrm{d}} \\
v_{0}=R i_{0}+\frac{\mathrm{d} \psi_{0}}{\mathrm{~d} t}
\end{gathered}
$$

The ohmic resistance of the stator windings is $R$ and the electric angular frequency is $\omega$. The voltage, flux-linkage and current components of the direct-axis and quadrature-axis are $\boldsymbol{v}_{x}, \boldsymbol{\psi}_{x}$ and $\boldsymbol{i}_{x}$ with $x \in\{\mathrm{d}, \mathrm{q}\}$, while $v_{0}, \psi_{0}$ and $i_{0}$ are the zerosequence components. As the sum of the phase voltages of delta-connected machines equals zero at every point in time, the voltage $v_{0}$ equals zero too (Kirchhoff's second law).

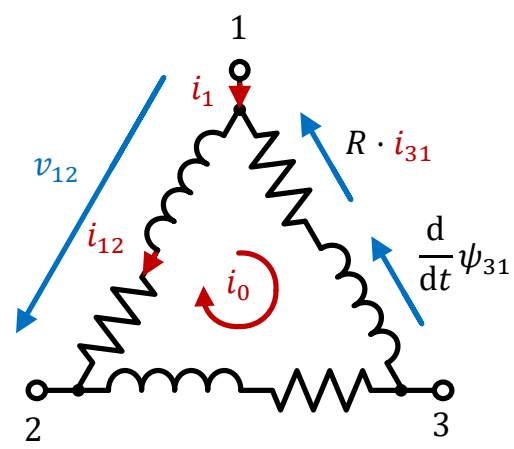

Fig. 2 Delta-connected PMSM with the zero-sequence current $i_{0}$, the phase current $i_{12}$ and the terminal current $i_{1}$.

The flux-linkages $\boldsymbol{\psi}_{\mathrm{dq} 0}$ can described, as also in [7] shown, as a function of $\boldsymbol{f}\left(i_{\mathrm{d}}, i_{\mathrm{q}}, \gamma, \omega\right)$ with $\gamma$ as the electric rotor angular. The zero-sequence flux-linkage is thereby assumed to be independent of the zero-sequence current because this current cannot be directly controlled. The machine voltage equations (1) to (3) can rewritten, considering the total derivative of the flux-linkages with the partial derivations, as derived in [8]. Constant currents and constant rotor speed yield to disappearing time-derivatives of the currents in the voltage equations and allow the simplification of the equations as seen in (6) and (7). Furthermore, the fluxlinkages and the stator resistance at steady-state operation allow the inner torque and zero-sequence current calculation, [7]. Due to this, the inner torque can be calculated considering the inner power of the machine and the power balance with the mechanical power, as described in [9]. The inner torque $T_{\mathrm{i}}$ is represented only through the time-variant rotor position flux-linkages and the currents, as shown in equation (4). Thereby $p$ denotes the number of pole pairs, the factor $3 / 2$ is due to the amplitude invariant transformation. As explained in $[6,10]$, the effects of cogging torque cannot be calculated properly with electrical quantities and are therefore neglected in the following. In equation (4), the term $\frac{3 p}{2}\left(\psi_{\mathrm{d}} i_{\mathrm{q}}-\psi_{\mathrm{q}} i_{\mathrm{d}}\right)$ describes the already known fundamental torque $T_{\mathrm{i}, \text { fnd. }}$. The terms $\frac{\partial \psi_{\mathrm{dq} 0}}{\partial \gamma} i_{\mathrm{dq} 0}$ supplement the fundamental equation [6], they are denoted as dynamic inner torque and describe the rotor angular position dependent inner torque of the machine.

$$
T_{\mathrm{i}}=\frac{3 p}{2}\left(\left(\psi_{\mathrm{d}} i_{\mathrm{q}}-\psi_{\mathrm{q}} i_{\mathrm{d}}\right)+\left(\frac{\partial \psi_{\mathrm{d}}}{\partial \gamma} i_{\mathrm{d}}+\frac{\partial \psi_{\mathrm{q}}}{\partial \gamma} i_{\mathrm{q}}+2 \frac{\partial \psi_{0}}{\partial \gamma} i_{0}\right)\right)
$$

The extended dq0-model enables also the zero-sequence current calculation. The evaluation of the partial derivation of the zero-flux-linkage at steady-state operation yields the zerosequence current, with $\omega=\partial \gamma / \mathrm{d} t$.

$$
i_{0}=-\frac{1}{R} \frac{\partial \psi_{0}}{\partial \gamma} \omega
$$

\section{PARAMETERIZATION APPROACH}

For the identification of the parameters, the dq-voltages and the zero-sequence current from testbench measurement and FEA simulation are evaluated. The flux-linkages $\boldsymbol{\psi}_{\mathrm{dq} 0}\left(i_{\mathrm{d},} i_{\mathrm{q}}, \gamma, \omega\right)$ at different constant rotor speeds are simplified to $\left.\boldsymbol{\psi}_{\mathrm{dq} 0}\left(i_{\mathrm{d}}, i_{\mathrm{q}}, \gamma\right)\right|_{\omega=\text { const. }}$.

\section{A. Stator Resistance}

The stator resistance can be measured directly. Assuming a balanced stator resistance and applying a constant DC-current $i_{\mathrm{DC}}$ with a DC-source at terminals ' 1 ' and ' 2 ' at defined thermal conditions yields the voltage drop $v_{12}$. Considering the machines ohmic voltage divider $R=\frac{3}{2} \frac{v_{12}}{i_{\mathrm{DC}}}$ yields the stator resistance of the model.

\section{B. DQ-Flux-Linkage}

Ideal constant dq-currents, at constant rotor speed enable, as motivated, the simplification of the voltage equations (1) to (3). Due to this, the time-dependent dq-currents vanish and the flux-linkage can be further simplified to

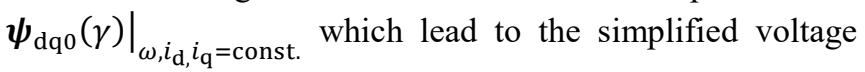
equations (6) and (7).

$$
\begin{aligned}
& v_{\mathrm{d}}=R i_{\mathrm{d}}+\frac{\partial \psi_{\mathrm{d}}}{\partial \gamma} \frac{\mathrm{d} \gamma}{\mathrm{d} t}-\omega \psi_{\mathrm{q}} i_{\mathrm{d}} \\
& v_{\mathrm{q}}=R i_{\mathrm{q}}+\frac{\partial \psi_{\mathrm{q}}}{\partial \gamma} \frac{\mathrm{d} \gamma}{\mathrm{d} t}+\omega \psi_{\mathrm{d}} i_{\mathrm{q}}
\end{aligned}
$$

For the solution of these coupled differential equations the "separation of the variables" method, as described in detail in [7], is used. Thereby the measurable voltage time-series can rewritten as a Fourier series as shown in equation (8), $\rho$ denotes thereby the order of the investigated harmonic frequency in the dq-reference frame.

$$
\boldsymbol{v}_{\mathrm{dq}}(t)=\frac{\overline{\boldsymbol{v}_{\mathrm{dq}}}}{2}+\sum_{\rho=1}^{\frac{N}{2}} \boldsymbol{v}_{\mathrm{dq}, \mathrm{a}, \rho} \cos (\omega \rho t)+\boldsymbol{v}_{\mathrm{dq}, \mathrm{b}, \rho} \sin (\omega \rho t)
$$

The general solution $y$ of the differential equation is assumed as $y=y_{\mathrm{c}}+y_{\mathrm{p}}$. Equation (9) denotes the particular integral $y_{\mathrm{p}}$ of the solution, which is the trivial solution of this differential equation. The variables of $y_{\mathrm{p}}$ are all time-invariant.

$$
\left(\begin{array}{l}
\bar{\psi}_{\mathrm{d}} \\
\bar{\psi}_{\mathrm{q}}
\end{array}\right)=\frac{1}{\omega}\left(\begin{array}{cc}
0 & 1 \\
-1 & 0
\end{array}\right) \cdot\left(\left(\begin{array}{l}
\bar{v}_{\mathrm{d}} \\
\bar{v}_{\mathrm{q}}
\end{array}\right)-R\left(\begin{array}{l}
i_{\mathrm{d}} \\
i_{\mathrm{q}}
\end{array}\right)\right)
$$

As a result of $y_{\mathrm{p}}$ the complementary function $y_{\mathrm{c}}$ includes 
only the time-variant terms (10), the current or stator resistance dependencies are vanished.

$$
\left(\begin{array}{l}
v_{\mathrm{d}, \rho}(t) \\
v_{\mathrm{q}, \rho}(t)
\end{array}\right)=\frac{\mathrm{d}}{\mathrm{d} t}\left(\begin{array}{l}
\psi_{\mathrm{d}, \rho}(t) \\
\psi_{\mathrm{q}, \rho}(t)
\end{array}\right)+\omega\left(\begin{array}{cc}
0 & -1 \\
1 & 0
\end{array}\right) \cdot\left(\begin{array}{l}
\psi_{\mathrm{d}, \rho}(t) \\
\psi_{\mathrm{q}, \rho}(t)
\end{array}\right)
$$

As the flux-linkages are the time-derivatives of the voltages, the flux-linkage harmonics are assumed as of the same order as the voltage harmonics. Insertion of both (voltage and flux-linkage) Fourier series, simplifies the complementary function (10) to equation (11). The Fourier series coefficients for the voltages are $\boldsymbol{v}_{\mathrm{dq}, \mathrm{ab}, \rho}$, as shown in (8). For the flux-linkages the coefficients are similar defined as $\boldsymbol{\psi}_{\mathrm{dq}, \mathrm{ab}, \rho}$.

The matrix $\boldsymbol{V}$ denotes all the voltage Fourier coefficients, the matrix $\boldsymbol{\psi}$ denotes all the flux-linkage Fourier coefficients. The matrix $\boldsymbol{F}$ describes a vector with $\left(\begin{array}{l}\cos (\omega \rho t) \\ \sin (\omega \rho t)\end{array}\right)$.

$$
\boldsymbol{V} \cdot \boldsymbol{F}(\omega \rho t)=\frac{\mathrm{d}}{\mathrm{d} t}(\boldsymbol{\psi} \cdot \boldsymbol{F}(\omega \rho t))+\omega\left(\begin{array}{cc}
0 & -1 \\
1 & 0
\end{array}\right) \boldsymbol{\psi} \cdot \boldsymbol{F}(\omega \rho t)
$$

After mathematical simplification, with using the method of equating coefficients for the system of linear equations (11), the Fourier series coefficients of the dq-flux-linkages can be calculated directly through the measured voltage coefficients, as displayed in (12).

$$
\begin{aligned}
\text { I. } & \psi_{\mathrm{d}, \mathrm{a}, \rho}=\frac{-v_{\mathrm{q}, \mathrm{a}, \rho}-\rho v_{\mathrm{d}, \mathrm{b}, \rho}}{\omega\left(\rho^{2}-1\right)} \\
\text { II. } & \psi_{\mathrm{d}, \mathrm{b}, \rho}=\frac{-v_{\mathrm{q}, \mathrm{b}, \rho}+\rho v_{\mathrm{d}, \mathrm{a}, \rho}}{\omega\left(\rho^{2}-1\right)} \\
\text { III. } & \psi_{\mathrm{q}, \mathrm{a}, \rho}=\frac{v_{\mathrm{d}, \mathrm{a}, \rho}-\rho v_{\mathrm{q}, \mathrm{b}, \rho}}{\omega\left(\rho^{2}-1\right)} \\
\text { IV. } & \psi_{\mathrm{q}, \mathrm{b}, \rho}=\frac{v_{\mathrm{d}, \mathrm{b}, \rho}+\rho v_{\mathrm{q}, \mathrm{a}, \rho}}{\omega\left(\rho^{2}-1\right)}
\end{aligned}
$$

The flux-linkage coefficients $\boldsymbol{\psi}_{\mathrm{dq}, \mathrm{ab}, \rho}$ of each $\rho$ describes the Fourier series of the flux-linkages $\boldsymbol{\psi}_{\mathrm{dq}}$ at constant rotor speed $\omega$ and constant currents $\boldsymbol{i}_{\mathrm{dq}}$. Evaluation of the Fourier series for each $\rho$ leads to the estimated flux-linkage timeseries $\boldsymbol{\psi}_{\mathrm{dq}}(t)$ with $\gamma(t)=\omega t$. The flux-linkage time-series can rewritten as $\left.\boldsymbol{\psi}_{\mathrm{dq}}\left(i_{\mathrm{d}}, i_{\mathrm{q}}, \gamma\right)\right|_{\omega=\text { const. }}$, [7].

\section{Zero-Sequence Flux-Linkage}

For zero-sequence flux-linkage parameterization terminal current sensors, measuring $i_{1,2,3}$ are not sufficient, additional phase current sensors, measuring $i_{12,23,31}$ are necessary. The zero-sequence current $i_{0}$ can calculated through (13).

$$
i_{0}=\frac{1}{3}\left(i_{12}+i_{23}+i_{31}\right)
$$

According equation (3) with $u_{0}=0 \mathrm{~V}$ for delta-connected PMSMs, the mathematical integration of the ohmic voltage drop of $i_{0}$ over the stator resistance $R$ results in the fluxlinkage $\psi_{0}\left(i_{\mathrm{d}}, i_{\mathrm{q}}, t\right)$ as shown in equation (14). As motivated before, $\psi_{0}\left(i_{\mathrm{d}}, i_{\mathrm{q}}, t\right)$ can be rewritten as $\left.\psi_{0}\left(i_{\mathrm{d}}, i_{\mathrm{q}}, \gamma\right)\right|_{\omega=\text { const. }}$.

$$
\int_{t_{0}}^{t_{1}} R i_{0} \mathrm{~d} t=\psi_{0}
$$

\section{DEVICE UNDER TEST}

\section{A. Requirements}

For the determination of the zero-sequence currents, control as well as precise and symmetrical measurement of the phase currents $i_{12,23,31}$ is mandatory. With purchasable delta-connected PMSM machines, measurement of these inner currents is difficult or even impossible. Therefore, a special DUT was created with a possible delta- and star- connection of the phases. The requirements for this machine differs from the typical design issues. The goal was the design of an easy manufacturable PMSM with visible iron saturation for valid zero-sequence currents and preferably symmetrical phase current measurement. Furthermore, the peak torque has to be smaller than $8.5 \mathrm{Nm}$ with a maximum power smaller than $1 \mathrm{~kW}$ because of the maximum quantities of the available testbench, inverters and load machine.

\section{B. Machine Design}

Due to the manual manufacturing process of the DUT PMSM, an available commercial stator and rotor lamination from Kienle + Spiess of the type KSPM 80/4.70 was selected. The DUT is built with distributed windings. The stator and rotor lamination is shown in Fig. 3 (a), the main properties of the design are listed in Table I.

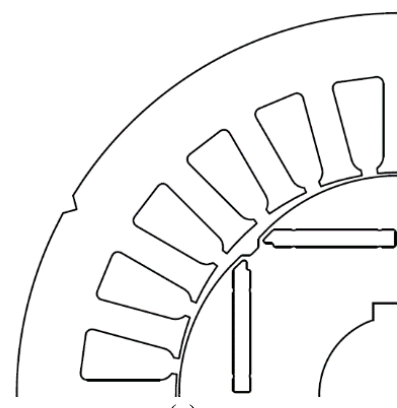

(a)

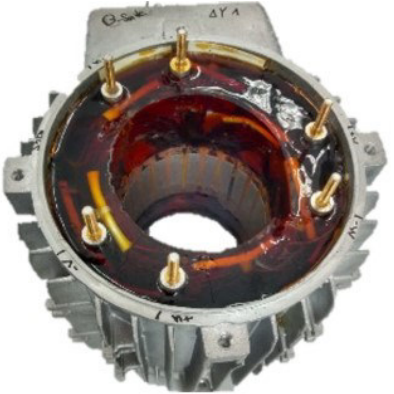

(b)
Fig. 3. (a) Quarter of the stator and rotor lamination of the DUT. (b) Stator body with connectors for assembly of a printed circuit board, encapsulated with epoxy resin.

The machine mechanics as body, bearing shields and the shaft are standardized parts. The stator body including the windings, the connectors for a Printed Circuit Board (PCB) and additional thermocouples are encapsulated with epoxy resin for thermal and mechanical linkage as shown in Fig. 3 (b). The rotor lamination with the magnetized $N d F e B$ magnets is mounted on a shaft. The machines design process, analytical calculations, winding placement was done equivalent as described in [9]. The windings are serially connected; the three phases are delta-connected, the phase connectors are accessible from the outside.

TABLE I

MAIN DESIGN PROPERTIES OF THE DEVICE UNDER TEST

\begin{tabular}{ccc}
\hline Quantities & Symbol & Value \\
\hline Num. of pole pairs & $p$ & 2 \\
Num. of phases & $m$ & 3 \\
Num. of slots per pole per phases & $q$ & 2 \\
Num. of winding turns & $N_{\mathrm{t}}$ & 23 \\
Num. of slots & $N_{\mathrm{s}}$ & 24 \\
Inner stator diameter & $D_{\mathrm{i}}$ & $70 \mathrm{~mm}$ \\
Outer stator diameter & $D_{\mathrm{o}}$ & $120 \mathrm{~mm}$ \\
Rotor diameter & $d_{\mathrm{i}}$ & $69 \mathrm{~mm}$ \\
Length & $L_{\mathrm{Fe}}$ & $30 \mathrm{~mm}$ \\
Wire diameter & $D_{w}$ & $1.5 \mathrm{~mm}$ \\
\hline
\end{tabular}

For the additional Finite Element Analysis (FEA) the software Flux 2D (Altair) was used. FEA and measurement results enable the comparison of both data for a detailed analysis. The main quantities for the measurement and the FEA are listed in Table II. The permanent magnet fluxlinkage of the FEA as well as the stator resistance was adopted from the testbench parameters for improved coherence. 
TABLE II

MAIN QUANTITIES OF THE DEVICE UNDER TEST

\begin{tabular}{ccc}
\multicolumn{3}{c}{ MAIN QUANTITIES OF THE DEVICE UNDER TEST } \\
\hline Quantities & Symbol & Value \\
\hline Maximum voltage & $v$ & $48 \mathrm{~V}$ \\
Maximum current & $i$ & $16 \mathrm{~A}$ \\
Nominal speed & $n$ & $1000 \mathrm{rpm}$ \\
Nominal torque & $T$ & $3.3 \mathrm{Nm}$ \\
Permanent magnet flux-linkage & $\psi_{\mathrm{PM}}$ & $70.1 \mathrm{mVs}$ \\
Stator resistance & $R$ & $413 \mathrm{~m} \Omega$ \\
\hline
\end{tabular}

\section{Phase Interconnection}

For the symmetrical and low impedance interconnection of the three phases, a PCB was designed. Each phase is thereby connected to the other via precise current sensors of type LEM CKSR-25NP. The PCB contains also shielded signal wiring and the power supply connectors, Fig. 4 (a). The manufactured and mounted PCB is shown in Fig. 4 (b).

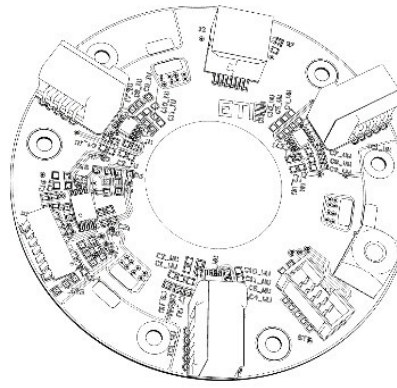

(a)

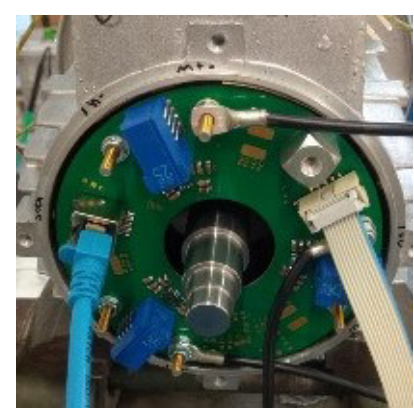

(b)
Fig. 4. (a) PCB interconnection with precise current measurement. (b) Mounted and wired PCB without outer bearing shield.

\section{EXPERIMENTAL SETUP}

\section{A. Power Electronics and Signal Processing System}

The back to back mounting of the DUT and the Load Machine (LM) is shown in Fig. 5 (b). The LM is a PMSM type Nanotec DB80C04803-ENM05J. The used voltage source inverters have a shared DC-link, supplied by a DC power supply. The inverter MOSFETs are of the type Texas Instrument CSD19535KCS with a switching frequency of $16 \mathrm{kHz}$. The currents for the control of the DUT are detected by the introduced phase current measurement of the DUT. The phase voltages are directly measured via precise voltage dividers. The sampling frequency of the ADCs, type Texas Instrument THS 1206 (12 bit), is $1.5 \mathrm{Msps}$. The rotor speed and the rotor position are determined with a Heidenhain ROC1013 13-bit encoder. The control for the LM and the DUT each, is done by a TMS320C6748 digital signal processor from Texas Instruments. The control period is $62.5 \mu \mathrm{s}$, according to the switching frequency of $16 \mathrm{kHz}$. The inverter switching signals are generated on a Cyclone 4 field programmable gate array from Altera/Intel. A more detailed view of the modular signal processing system can be found in [11]. The whole power electronics and signal processing system cabinet is shown in Fig. Fig. 5(a).

\section{B. Control Algorithm and Parameter Measurement}

The LM is speed controlled by a standard PI type control algorithm. The DUT is controlled by a specially designed control algorithm for nonlinear machines [2], with additional mitigation of the current harmonics. The implementation of the control algorithm and the block diagram is adopted from [12]. This control algorithm enables nearly ideal constant dq-currents on the testbench as required for parameterization approach. For the initial fundamental flux- linkage lookup tables of the controller [2] the estimated FEA flux-linkages are used. For the testbench parameter estimation of the flux-linkages, various operating points with evenly distributed $\left(i_{\mathrm{d}}, i_{\mathrm{q}}\right)$ currents from $-16 \mathrm{~A}$ to $16 \mathrm{~A}$, constant speed $\omega=1000 \mathrm{rpm}$ and constant temperatures $\vartheta=40^{\circ} \mathrm{C}$ are automatically applied by a National Instruments LabVIEW routine. The quantities of interest are measured with the introduced signal processing system.

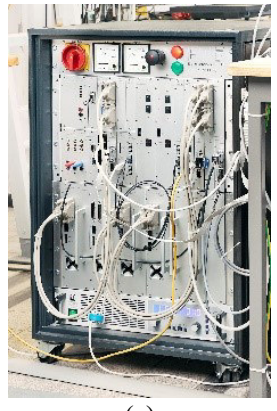

(a)

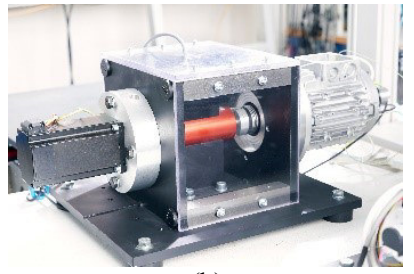

(b)
Fig. 5. (a) Testbench cabinet with inverters and signal processing, (b) mechanical assembly of DUT and LM.

\section{RESULTS}

In the following section the identification of the main model parameters $R$ and $\left.\boldsymbol{\psi}_{\mathrm{dq} 0}\left(i_{\mathrm{d}}, i_{\mathrm{q}}, \gamma\right)\right|_{\omega=\text { const. }}$, for the parameter lookup table, is described. The flux-linkages planes are visualized for $\omega=$ const., at one defined rotor position. For the rotor position dependencies the time-response of the flux-linkages, the zero-sequence current and the inner torque for an exemplarily operation point with $i_{\mathrm{d}}, i_{\mathrm{q}}, \omega=$ const., is shown.

\section{A. Stator Resistance Parameterization}

Applying a constant DC-current of $10 \mathrm{~A}$ at $\vartheta=40^{\circ} \mathrm{C}$ leads to a stator resistance of $R=0.4125 \Omega$. The theoretical stator resistance, calculated by the material resistivity and the length is $R_{\text {ideal }}=0.0556 \Omega$. The significant difference is due to the contact resistances, the interconnection of the windings, the $\mathrm{PCB}$ and the additional current sensors within the phases.

\section{B. DQ-Flux-Linkage Parameterization}

\section{1) Time-Variant DQ-Flux-Linkages}

At stationary operation the flux-linkages are still timevariant since they depend on the rotor angular position. Fig. 6 draft the principle of the dq-flux-linkage parameterization based on measureable voltages, exemplarily with FEA generated data for a better visibility and interpretation. In Fig. 6 (f), the dq-flux-linkages, which have to be identified are displayed blue and green. The corresponding and measureable dq-voltages are shown in the subfigure (a), also in green and blue. The dominant flux-linkage harmonics are the sixth and twelfth, [9]. Then, also the dq-voltages show the sixth and twelfth harmonics, but in different amplitudes and phasing. The parameterization approach from [7], is used to calculate the solution of the differential equation $y=y_{\mathrm{c}}+y_{\mathrm{p}}$. Thereby, the particular solution $y_{\mathrm{p}}$ is calculated with the dqmean-voltage and the stator resistance. The complementary solution $y_{c}$ is reconstructed by permutation of the $\mathrm{d}$ - and q- axis Fourier voltage coefficients and their factorization with their harmonic $\rho$. As an example, the d-axis-voltage Fourier coefficients $v_{\mathrm{d}, \mathrm{ab}, \rho}$ are shown in Fig. $6(\mathrm{~b}, \mathrm{c})$. The green crosses in (a), show thereby the d-axis voltage timeseries of the Fourier coefficients for the Fourier analysis validation. The calculated q-axis-flux-linkage Fourier 
coefficients are shown in (d) and (e). Due to ideal conditions in the FEA simulation only the expected harmonics of the $6^{\text {th }}$ and $12^{\text {th }}$ of the flux-linkages and voltages are visible [9]. In (f) the flux-linkage time-series, based on this Fourier coefficients, is drawn as blue circles. The directly FEA calculated q-flux-linkage is shown as blue line, both results are similar to each other.

(a)
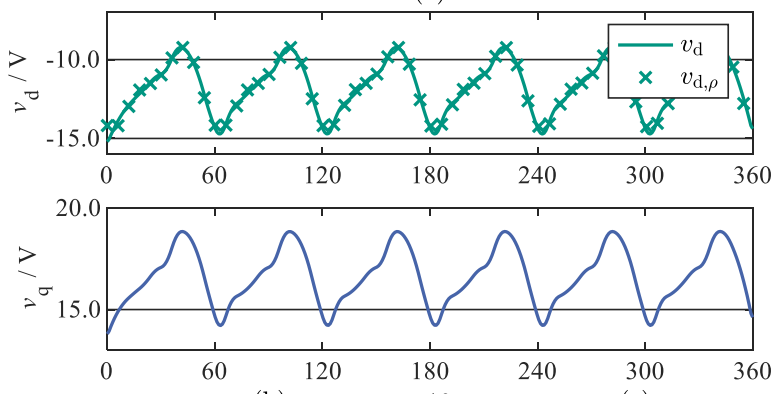

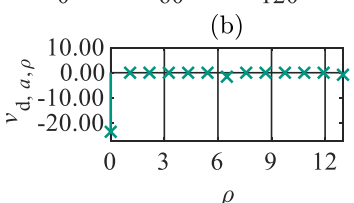

$(\mathrm{d})$
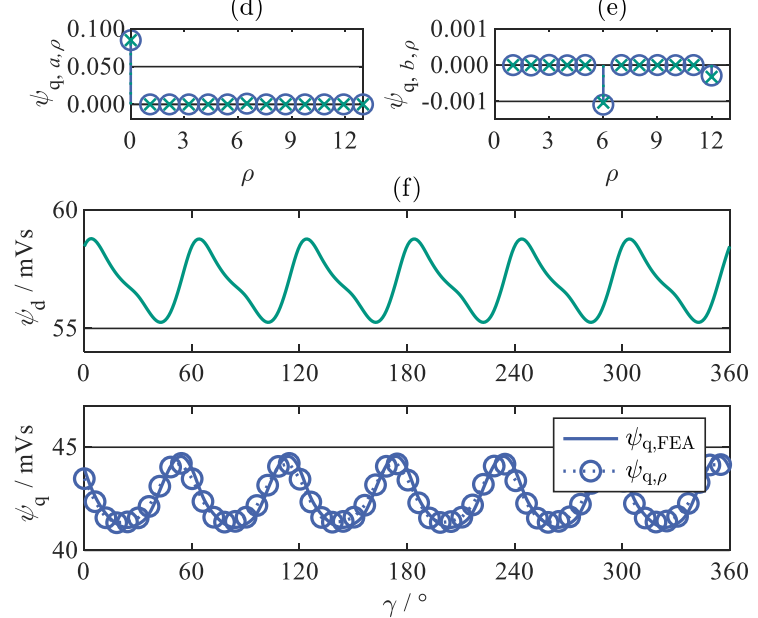

Fig. 6 (a) FEA calculated d- and q-axis voltages with the Fourier approximated time-series of the d-axis-voltage. $(b, c)$ describes the Fourier coefficients of the $d$-axis voltage. The Fourier coefficients after reconstruction of the q-flux-linkage are (d, e). (f) shows the FEA calculated dq-flux-linkages. Additionally, the time-series of the parameterized position dependent q-flux-linkages is drawn.

Fig. 7 shows the FEA, the estimated and the with the mean values corrected rotor position dependent dq-flux-linkages at constant rotor speed and currents. Thereby the FEA calculated dq-values are green and blue, the estimated flux-linkages are marked with gray dots. The black dashed line is only for visibility, without the mean value error: $\boldsymbol{\psi}_{\mathrm{dq}, \text { corr }}=\overline{\boldsymbol{\psi}}_{\mathrm{dq}, \mathrm{FEA}}-$ $\overline{\boldsymbol{\psi}}_{\text {dq,MEAS }}+\boldsymbol{\psi}_{\text {dq,MEAs }}$. Comparing the FEA simulated dq-flux-linkages and the estimated dq-flux-linkages, the harmonic frequency behavior and the amplitudes of both look similar even with the slight phasing error. The mean value error $\overline{\boldsymbol{\psi}}_{\mathrm{dq}, \mathrm{FEA}}-\overline{\boldsymbol{\psi}}_{\mathrm{dq}, \mathrm{MEAS}}$ is thereby larger compared to the mismatch of the harmonics. There could be many reasons for this error. Reasons are, the neglected effect of the manufacturing process which influences the simulation results. Further, the iron losses are not considered in the FEA but visible in the measurement results. For comparability of both, without extensive test-bench iron loss characterization, the measurement is done at relatively low rotor speed for a small impact. Also a non-ideal relation of stator diameter and length had to be chosen due to the testbench requirements, especially with the available 2D FEA the coil end effects are not covered and yield to an additional mismatch. Additionally, the permanent magnet parameters as well as the iron parameter $\mathrm{B}(\mathrm{H})$ curve were only assumed as datasheet parameters and the rotor/stator geometry was retraced. Even with disregarding all of these effects in the simulation and measurement, the flux-linkages of both yield to comparable results. The harmonic behavior relies on the geometrical design of the slotting of the machine, etc. and behave similar results for measurement and calculation.
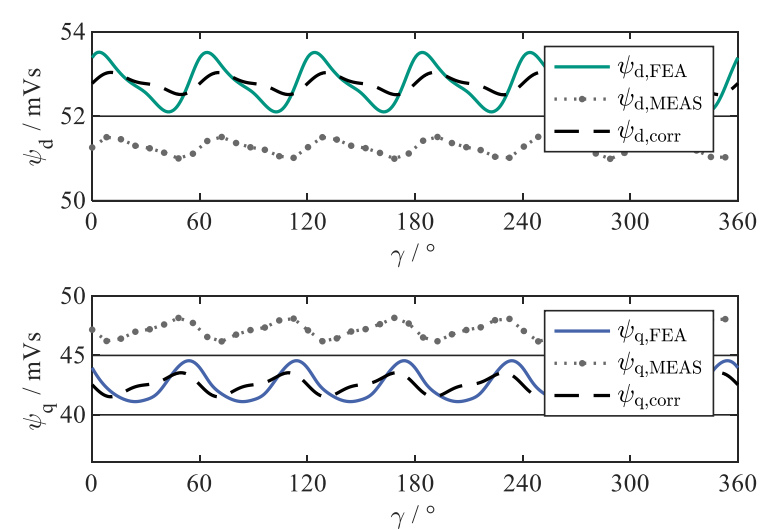

Fig. 7 FEA simulated, testbench estimated and the corrected testbench estimated rotor position dependent dq-flux-linkages at $n=1000 \mathrm{rpm}$ with $i_{\mathrm{d}}=-7 \mathrm{~A}$ and $i_{\mathrm{q}}=11.5 \mathrm{~A}$.

\section{2) DQ-Flux-Linkage Planes}

In Fig. 8 the flux-linkages $\left.\boldsymbol{\psi}_{\mathrm{dq}}\left(i_{\mathrm{d}}, i_{\mathrm{q}}\right)\right|_{\omega, \gamma=\text { const. }}$ are shown. Thereby the FEA flux-linkage plane is the colored surface, the testbench estimated flux-linkage plane is the gray meshed plane. (a)

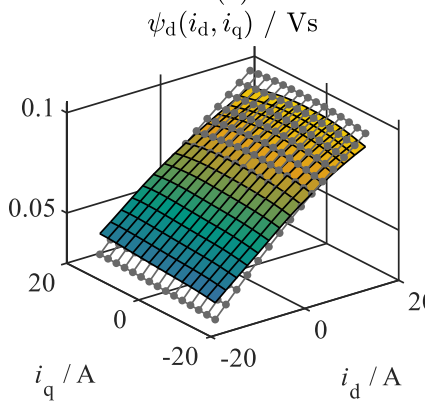

(b)

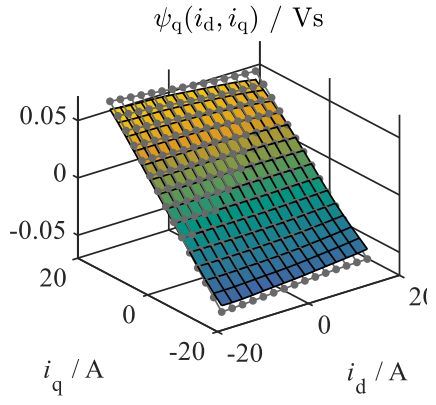

Fig. 8 (a) d-flux-linkage and (b) q-flux-linkage plane at $\gamma=60^{\circ}$ and $n=1000 \mathrm{rpm}$. The colored surface is thereby the FEA generated result, the gray mesh is the corresponding estimated data.

The supporting points of the displayed planes are the constant dq-currents $i_{\mathrm{d}}$ and $i_{\mathrm{q}}$. There are flux-linkage planes for each rotor position $\gamma$ at constant rotor speed $\omega$ as derived in Fig. 6. Iron saturation and cross-coupling effects are visible in the estimated and simulated flux-linkage planes. The difference between the flux-linkage planes from the measurement and the simulated data in the $\mathrm{d}$ - and q-axis is at least about $10 \%$. Possible reasons were discussed before.

\section{Zero-Sequence Flux-Linkage Parameterization}

1) Zero-Sequence Current

In Fig. 9, the zero-sequence current of an operating point at constant dq-currents and constant rotor speed is shown. The 
$\mathrm{x}$-axis shows the angular rotor position from $0^{\circ}$ to $360^{\circ}$, with $\gamma(t)=\omega t$. The rotor position dependent zero-sequence current $i_{0}$ from the FEA simulation is drawn red, the testbench measured current is shown as gray dotted line. The corresponding dq-currents are blue and green. As described in [9], the main harmonic of the zero-sequence current, can be identified as a multiple of a third harmonic in relation to the fundamental frequency. Thereby the amplitude and the phase of the zero-sequence current is dependent on the operating point, in this case at $\sqrt{ }\left(i_{\mathrm{d}}^{2}+i_{\mathrm{q}}^{2}\right)=13.5 \mathrm{~A}$. The peak-to-peak $(p-p)$ value of the zero-sequence-current in the FEA simulation is approx. $i_{0, \mathrm{FEA}, \mathrm{p}-\mathrm{p}}=5.5 \mathrm{~A}$. The value of the measurement is $i_{0, \mathrm{MEAS}, \mathrm{p}-\mathrm{p}}=5.2 \mathrm{~A}$. Reasons for the difference, are imprecise parameters for the FEA simulations as discussed before. Further factors which influence particular the zero-sequence current are detailed investigated in [13]. For the testbench zero-sequence flux-linkage identification, the measurement of the current $i_{0}$ is mandatory.

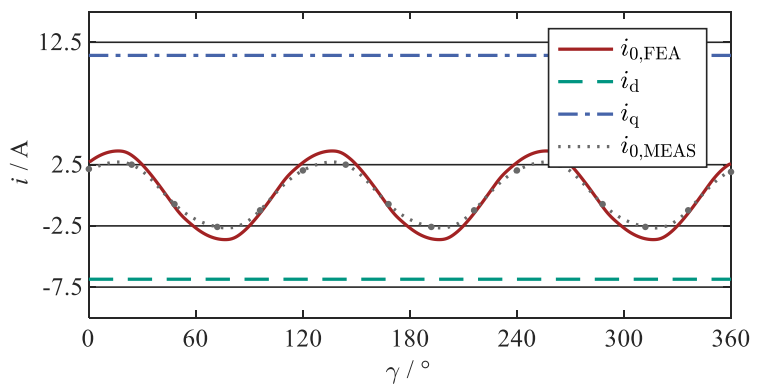

Fig. 9 Rotor position dependent FEA calculated current $i_{0, \mathrm{FEA}}$ and testbench measured current $i_{0, \text { MEAS }}$ is displayed at $n=1000 \mathrm{rpm}$ at $i_{\mathrm{d}}=-7 \mathrm{~A}$ and $i_{\mathrm{q}}=11.5 \mathrm{~A}$.

\section{2) Time-Variant Zero-Sequence Flux-Linkage}

Fig. 10 shows the zero-sequence flux-linkage of an operating point at constant dq-currents and constant rotor speed. The $\mathrm{x}$-axis denotes also one electric rotor angular from $0^{\circ}$ to $360^{\circ}$.

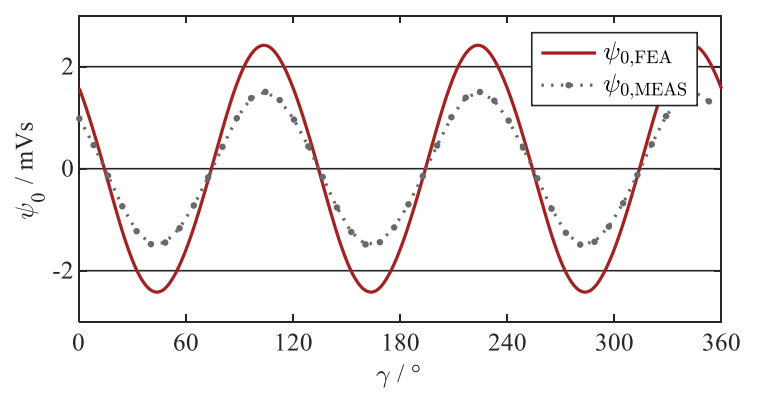

Fig. 10 Rotor position dependent FEA calculated zero-sequence flux-linkage $\psi_{0, \mathrm{FEA}}$ and the testbench estimated zero-sequence flux-linkage $\psi_{0, \mathrm{MEAS}}$ at $n=1000 \mathrm{rpm}$ at $i_{\mathrm{d}}=-7 \mathrm{~A}$ and $i_{\mathrm{q}}=11.5 \mathrm{~A}$.

The rotor position dependent flux-linkage $\psi_{0}$ from the FEA simulation is red drawn, the measurement result is shown as gray dotted line. For the calculation of $\psi_{0}$ as shown in equation (14), the measured current and a precise determined stator resistance is necessary. The integration of the voltage drop of the zero-sequence current at the stator resistance is thereby sensitive to stator resistance parameter variations and inaccuracy of the current measurements. Despite supposed parameter mismatches of the FEA and the measurement results, both results look similar and enable further calculations based on these quantities.

\section{3) Zero-Sequence Flux-Linkage Plane}

Fig. 11 shows the flux-linkage plane $\left.\psi_{0}\left(i_{\mathrm{d}}, i_{\mathrm{q}}\right)\right|_{\omega, \gamma=\text { const. }}$. The FEA calculated flux-linkage plane is the colored surface, the testbench estimated flux-linkage plane is the gray meshed plane. The supporting points of the displayed plane are the dq-currents $i_{\mathrm{dq}}$. There is a flux-linkage plane for each rotor position $\gamma$ at constant rotor speed $\omega$. The difference between the flux-linkage planes from the measurement and the simulated data is at least about $16 \%$. As before discussed, the reasons for the difference are material data, geometrical tolerances, etc. Compared to the dq-flux-linkages as smooth surface, the zero-sequence flux-linkage plane appears with three raisings due to local iron saturation of the resulting nonideal three phase system. During machine operation these flux-linkage raisings yield a compensating current due to Lenz's law. These zero-sequence current lowers the zero sequence flux-linkage and therefore the iron saturation level of the machine. This also influences the behavior of the d- and q-axis flux-linkages.

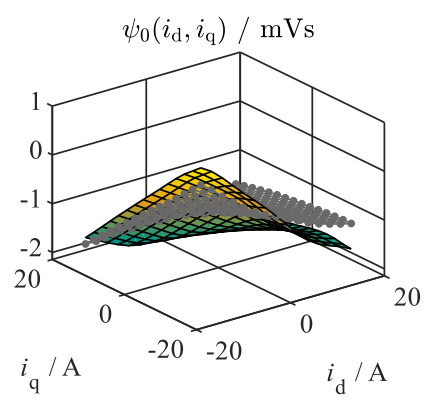

Fig. 11 Zero-Sequence flux-linkage plane at $\gamma=60^{\circ}$ and $n=1000 \mathrm{rpm}$. The colored surface is thereby the FEA generated result, the gray mesh is the corresponding estimated data.

\section{PMSM Inner Torque}

In Fig. 12 the theoretical torques of the DUT are shown. The estimated inner torque $T_{\mathrm{i} \text {,MEAS }}$ calculated with (4) is the gray dotted line. The FEA inner torque $T_{\mathrm{i}, \mathrm{FEA}}$, also calculated with the simulated flux-linkages and the currents is the purple line. As described in [6], an additional torque is effective due to the stray fluxes within stator and rotor, without coupling into the identifiable flux-linkages within the electric domain. The characterization of cogging torque is challenging [6] and needs extended measurements which is not part of this paper. Nevertheless, in FEA this term can be determined and is shown as $T_{\mathrm{FEA}}$ in the figure. In the FEA, torque calculation is usually based on virtual displacement methods [5], these methods allow the full magnetic torque calculation including the cogging torque. Considering the cogging torque as difference of the inner torque and the FEA torque, it can be written as $T_{\mathrm{COG}}=T_{\mathrm{FEA}}-T_{\mathrm{i}, \mathrm{FEA}}$.

The motivated inner torque representation $T_{\mathrm{i}, \mathrm{FEA}}$ is thereby an approximation, closer to the real torque $T_{\mathrm{FEA}}$, compared to the state of the art fundamental and constant torque representations. This torque is thereby only dependent on the identifiable flux-linkages. The differences between the inner torque of the FEA and the measurement are due to the differences of the identified flux-linkages as described above. However, the mean value of the torque and especially the occurring harmonic frequencies and their phasing show correct behavior in the FEA and the measurement which allows a more precise machine torque description for further applications. 


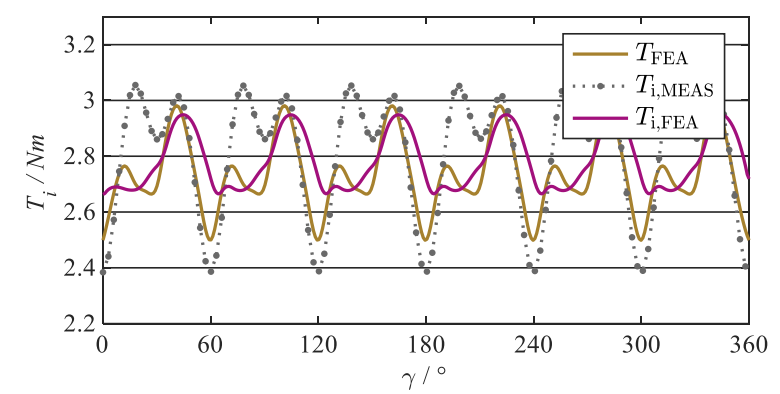

Fig. 12 Rotor position dependent FEA torque $T_{\mathrm{i}, \mathrm{FEA}}$, inner FEA torque $T_{\mathrm{i}, \mathrm{FEA}}$ and the testbench estimated inner torque $T_{\mathrm{i}, \mathrm{MEAS}}$ at $n=1000 \mathrm{rpm}$ and $i_{\mathrm{d}}=-7 \mathrm{~A}$ and $i_{\mathrm{q}}=11.5 \mathrm{~A}$.

\section{CONCLUSION}

The rotor position and rotor speed dependent dq0-fluxlinkages and the stator resistance, describe a dq0-model for delta-connected PMSMs. Usually FEA or extended analytical data is used for parameterization of these models. But since these data is always just an approximation, testbench measurements are desired. There are several theoretical parameterization approaches published in the past, but the time-variant flux-linkage calculation was still a challenging task. This paper shows a suitable testbench parameterization approach for the flux-linkages at steady-state testing. For the validation a self-build DUT PMSM is presented. The additional phase current sensing and the fast repetitive control algorithms at the testbench operation in combination with the introduced parameter identification scheme according [7] enables the full parameter identification. It can be shown that it is possible to parameterize rotor position and rotor speed depended flux-linkages for the introduced extended $\mathrm{dq} 0$ model. Furthermore, a comparison between the FEA and the measurement data is shown and the differences are discussed. The extended, testbench parameterized model can be used for advanced control algorithms with mitigation of the stationary inner torque ripple or sensorless control methods. Also a more precise system simulation is feasible, considering the zerosequence current losses in the thermal domain calculations based on this precise estimated data.

\section{REFERENCES}

[1] Pinto, D.E., Pop, A.-C., Kempkes, J., Gyselinck, J.: ‘dq0-modeling of interior permanent-magnet synchronous machines for high-fidelity model order reduction'. 2017 International Conference on Optimization of Electrical and Electronic Equipment (OPTIM) \& 2017 Intl Aegean Conference on Electrical Machines and Power Electronics (ACEMP), Brasov, Romania, May 25-27, 2017, pp. 357-363

[2] Richter, J., Doppelbauer, M.: 'Predictive Trajectory Control of Permanent-Magnet Synchronous Machines With Nonlinear Magnetics', IEEE Trans. Ind. Electron., 2016, 63, (6), pp. 3915-3924

[3] Rehm, F., Breining, P., Decker, S., Kolb, J., Hiller, M.: 'Loss Comparison of Small Delta- and Star-Connected Permanent Magnet Synchronous Machines'. 45th Annual Conference of the IEEE Industrial Electronics Society (IECON 2019), Lissabon, Portugal, pp. $1-10$

[4] Briz, F., Degner, M.: 'Rotor Position Estimation', EEE Ind. Electron. Mag., 2011, 5, (2), pp. 24-36

[5] Wang, X., Yi, P., Zhou, Z., Sun, Z., Ruan, W.: 'Improvements in the permanent magnet synchronous motor torque model using incrementa inductance', IET Electric Power Applications, 2020, 14, (1), pp. 109118

[6] Štumberger, Marčič: 'Magnetically Nonlinear Dynamic Models of Synchronous Machines and Experimental Methods for Determining their Parameters', Energies, 2019, 12, (18), p. 3519

[7] Decker, S., Rollbühler, C., Rehm, F., et al.: 'DQ0-Modelling and parametrization approaches for small delta connected permanent magnet synchronous machines'. 10th International Conference on
Power Electronics, Machines and Drives (PEMD 2020), Nottingham, England, 1-6 (accepted 2019-11-06, postponed to Dec'20 due to COVID-19)

[8] Stumberger, B., Stumberger, G., Dolinar, D., Hamler, A., Trlep, M.: 'Evaluation of saturation and cross-magnetization effects in interior permanent-magnet synchronous motor', IEEE Trans. on Ind. Applicat., 2003, 39, (5), pp. 1264-1271

[9] Hendershot, J.a.R., Miller, T.J.E.: 'Design of brushless permanentmagnet machines' (Motor Design Books, Venice, Fla., 2010)

[10] Krause, P.C., Wasynczuk, O., Sudhoff, S.D.: 'Analysis of electric machinery and drive systems' (IEEE Press Wiley-Interscience; IEEE Xplore, New York, Hoboken, NJ, Piscataway, New Jersey, 2002)

[11] Axtmann, C., Boxriker, M., Braun, M.: 'A custom, high-performance real time measurement and control system for arbitrary power electronic systems in academic research and education'. 2016 18th European Conference on Power Electronics and Applications (EPE'16 ECCE Europe), Karlsruhe, Sep. 05-09, 2016, pp. 1-7

[12] Richter, J., Doppelbauer, M.: 'Control and mitigation of current harmonics in inverter-fed permanent magnet synchronous machines with non-linear magnetics', IET Power Electronics, 2016, 9, (10), pp. 2019-2026

[13] Dinca, C., Schäfer, U.: 'Circulating currents of delta connected fractional slot machines for mass production', Elektrotech. Inftech., 2015, 132, (1), pp. 68-74

\section{BIOGRAPHIES}

Simon Decker was born in Oberkirch, Germany in 1988. He received the M.Sc. degree in electrical engineering from the Karlsruhe Institute of Technology (KIT) in 2015. Since 2015 he has been working as a research associate at the Institute of Electrical Engineering at KIT to receive a PhD. His research interests include power electronics and electrical drives, especially modelling, online identification and control strategies.

Simon Foitzik was born in Ellwangen, Germany in 1993. He received the M.Sc. degree in electrical engineering from the Karlsruhe Institute of Technology (KIT) in 2017. Since 2017 he has been working as a research associate at the Institute of Electrical Engineering at KIT to receive a PhD.

Felix Rehm was born in Stuttgart, Germany in 1992. He received the M.Sc. degree in electrical engineering from the Karlsruhe Institute of Technology (KIT) in 2018. Since 2018 he has been working as a research associate at the Institute of Electrical Engineering at KIT to receive a PhD.

Matthias Brodatzki was born in Bytom, Poland in 1988. He received the M.Sc. degree in electrical engineering from the Karlsruhe Institute of Technology (KIT) in 2014. Since 2014 he has been working as a research associate at the Institute of Electrical Engineering at KIT to receive a $\mathrm{PhD}$.

Christoph Rollbühler was born in Crailsheim, Germany in 1989. He received the M.Sc. degree in electrical engineering from the Karlsruhe Institute of Technology (KIT) in 2015. Since 2015 he has been working as a research associate at the Institute of Electrical Engineering at KIT to receive a PhD.

Johannes Kolb was born in Pforzheim, Germany, in 1982. He received his Dipl.-Ing. degree in electrical engineering from the University of Karlsruhe in 2007. From 2007 to 2013 he has been with the Institute of Electrical Engineering at the Karlsruhe Institute of Technology (KIT) as research associate in the field of power electronics, where he received his Dr.-Ing. in 2013. Since then he is leading a research team in the field of electric drives and power electronics at the SHARE at KIT - a cooperation between Schaeffler Technologies AG \& Co. KG and KIT.

Michael Braun received the Dipl.-Ing. and Dr.-Ing. degrees from the Technische Hochschule Darmstadt, Germany, in 1978 and 1983, respectively. From 1983 to 1994, he was with Siemens AG, Erlangen, Germany, where he was engaged in the development of power electronics and electrical drives. Since 1994, he has been a Professor and the Head of the Institute of Electrical Engineering, Karlsruhe Institute of Technology, Germany. His research interests include power electronics, control of electrical drives, and mechatronics. 